\title{
Wear of Si Cantilever Tips used in Atomic Force Acoustic Microscopy
}

\author{
R.H. Geiss, M. Kopycinska-Müller and D.C. Hurley \\ Materials Reliability Division \\ National Institute of Standards and Technology \\ 325 Broadway, Boulder, CO 80305-3328 USA
}

As the implementation of thin films continues to extend into the nanoscale, innovative experimental techniques are needed to study their physical properties. Many applications, for example molecular dynamics modeling, require knowledge about the mechanical properties of such nanoscale thin films. Unfortunately, most conventional methods to evaluate thin-film mechanical properties are illequipped to cope as film dimensions shrink below a micrometer. Recently, the efforts of several groups have focused on the determination of elastic properties using dynamic enhancements of atomic force microscopy (AFM) [1-4]. Despite individual differences, all of these methods rely on simplified Hertz or Derjaguin-Müller-Toporov (DMT) contact-mechanics models [5,6] to describe the interaction between the AFM tip and the sample. Atomic force acoustic microscopy (AFAM) $[3,4]$ has been used to obtain quantitative elastic-property values that agree with those obtained by nanoindentation or calculated from tabulated data. However, the best agreement is obtained when AFAM experiments include tedious reference measurements to minimize the influence of continuous tip wear. In this paper, we describe our work to better understand the process of tip wear that occurs in AFAM experiments in order to improve contact-mechanics models for data analysis.

Several silicon AFM cantilevers were used to conduct a sequence of tests that simulated typical AFAM experiments. AFAM experiments measure the resonant frequency of the cantilever when it is in contact with a vibrating sample as a function of the applied static load. The first test consisted of three AFAM measurements at relatively low loads (about 0.3 to $1.5 \mu \mathrm{N}$ ). In the second test, the static load was increased to $3-5 \mu \mathrm{N}$ and to as many as 40 repeat measurements. A fused quartz sample was used for all of the experiments. From the measured frequencies of the lowest two flexural modes, the contact stiffness $k^{*}$ was calculated [4]. The Hertz and DMT contact-mechanics models were used to estimate the geometry of the tip and the tip radius $R$ assuming a hemispherical-shaped tip. We also acquired scanning electron microscope (SEM) images of the tips at various points in the AFAM testing sequence and measured the apparent tip radius $R_{S E M}$. The values of $R_{S E M}$ were compared with the values of $R$ obtained from the AFAM measurements using the above-mentioned theories.

The details of the SEM-AFAM experiments and measurement results will be described. In many cases, the tip broke during the first AFAM measurement sequence and increased its radius during subsequent measurements, as seen in Figure 1. We found that the tips always changed. Some tips were found to have ill-defined geometries, as shown in Figure 2, but still yielded useful AFAM data. Broken tips quite frequently show a structure similar to the flat-punch geometry shown in Figure $2 \mathrm{c}$. We observed that tips with initial probe diameters of about $50 \mathrm{~nm}$ usually did not break and were the most robust throughout the AFAM measurement sequence. Tips with smaller probe diameters frequently broke in the first, low force loading. Larger tips give more erratic results. We have also examined a few tips in the transmission electron microscope (TEM) after the full AFAM test sequence and usually find a thin $(\sim 10 \mathrm{~nm})$ layer of amorphous material forms after tip use.

Dislocation arrays have also been seen originating at the tip in some of the tips following testing. The observation of a thin amorphous layer on Si cantilever tips has been previously reported [7]. 


\section{REFERENCES}

[1] F. Dinnelli et al., Phil. Mag. A 80, 2299 (2000).

[2] K. Yamanaka et al., Appl. Phys. Lett. 78, 1939 (2001).

[3] U. Rabe et al., Surf. Interface Anal. 33, 65 (2002).

[4] D. C. Hurley et al., J. Appl. Phys. 94, 2347 (2003).

[5] K. L. Johnson, Contact Mechanics (Cambridge University Press, 1985).

[6] B. Cappella and G. Dietler, Surf. Sci. Rep. 34, 1 (1999).

[7] K.-H. Chung et al., Ultramicroscopy 102, 161 (2005
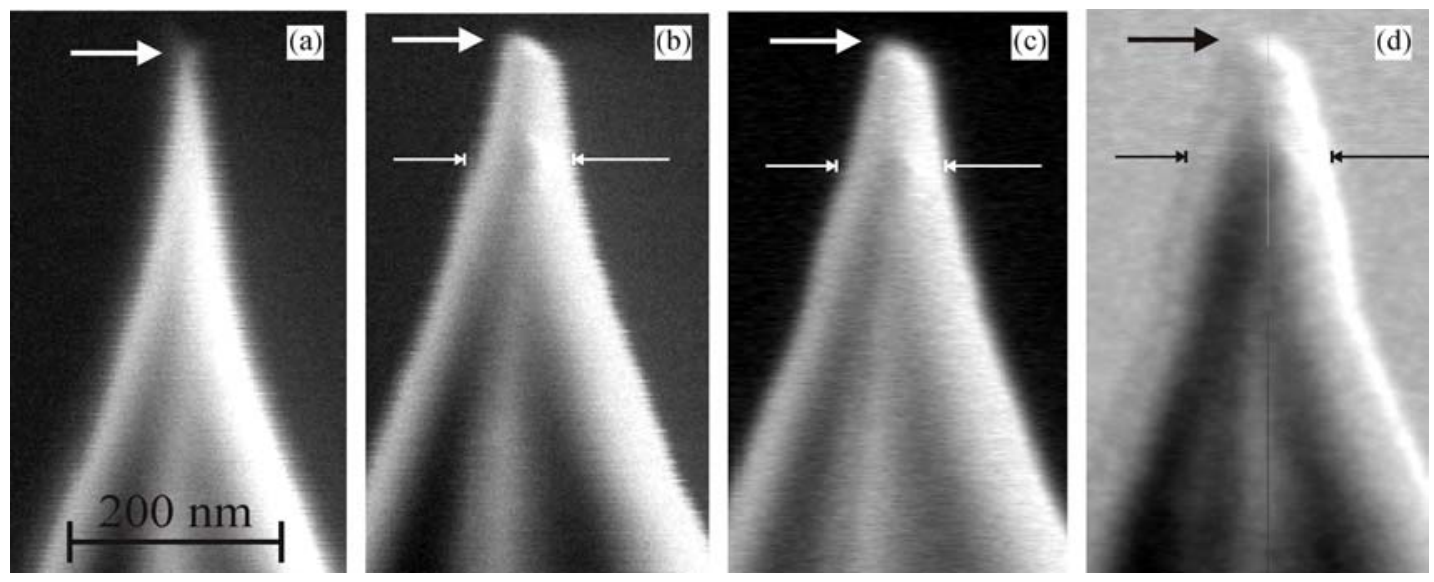

Figure 1. SEM images of an AFM tip. The scale, indicated in (a), is the same for all of the images. The arrows show where the tip changes were the largest. (a) New tip with $R<10 \mathrm{~nm}$. (b) Tip after the first AFAM test. The end of the tip has broken off, increasing $R$. (c) Tip after the second AFAM test. There is little change from (b), consistent with the AFAM results. (d) Tip after third AFAM test. Further increase in $R$ and changes in the tip width [as marked by pairs of arrows in (b), (c) and (d)] can be observed.
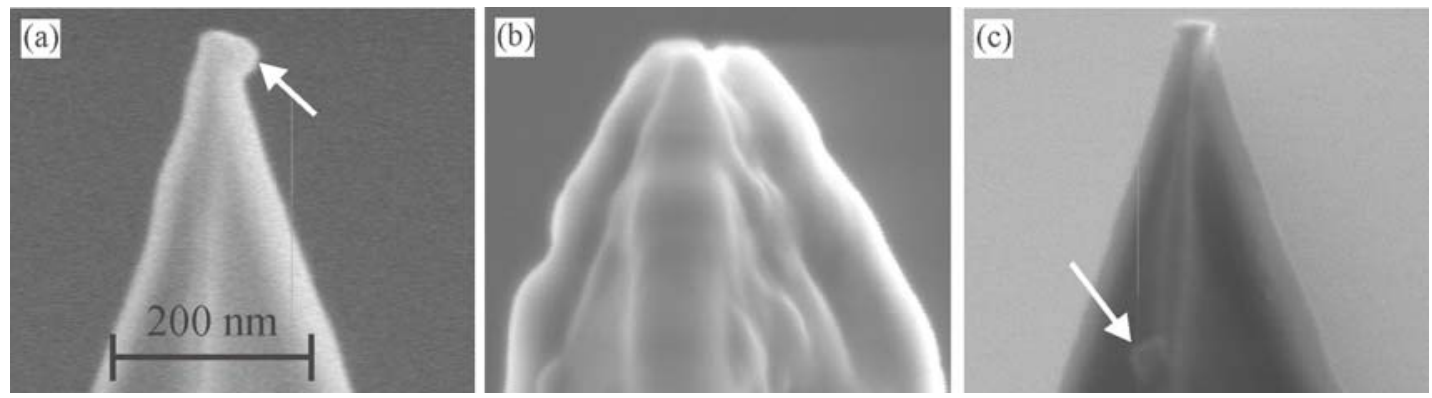

Figure 2. SEM images of three different AFM tips. The scale, indicated in (a), is the same for all of the images. Despite their dissimilar geometries, all of the tips yielded AFAM data that suggested hemispherical tip shapes. (a) Despite tip fracture, the tip is still relatively sharp and approximately round at the end. (b) The geometry of this tip is complex in comparison to the other tips. The tip shape changed dramatically during the experiments. (c) Example of flat-punch geometry. The arrow shows a particle of an unknown origin that contaminated the tip during the experiments. 